\title{
A Study on the Network Resource Openness with Software Networking toward the Development of the Web Technology
}

\author{
Jinhong Yang ${ }^{1}$, Hyojin Park ${ }^{2}$ and Jun Kyun Choi ${ }^{2}$ \\ ${ }^{1}$ Information and Communications Department, Korea Advanced Institute \\ of Science and Technology, 291, Daehak-ro, Yuseong-gu, Daejeon 34141 \\ ${ }^{2}$ School of Electrical Engineering, KAIST, Republic of Korea \\ E-Mail: \{jinhong.yang; gaiaphj\}@kaist.ac.kr; jkchoi@ee.kaist.ac.kr
}

Received 22 January 2016; Accepted 2 March 2016;

Published 15 March 2016

\begin{abstract}
The continuous development of the network and the growth of the Internet and the Web are festinating. The improvement in network softwarization technologies represented by the SDN and NFV made the dedicated legacy network resources into more elastic to be utilized. These progresses realize the accessibility on the customized network resource utilization for web services, overcoming traditional best effort network environment. HTTP based application services are extended from multimedia to IoT with fast evolution of Web technology. In this study, network resource open trends are explored in softwarization networking technology perspective, and describe what will be the future of network environment, step-by-step. Especially, the process of change is focused from previous individual communication service environment such as voice, data to unified communication environment.
\end{abstract}

This work was supported by Institute for Information \& communications Technology Promotion (IITP) grant funded by the Korea government (MSIP) (No. B0101-15-0331, Development of Multi-Screen Adaptive Autonomous Smart Media Technology)

Journal of Software Networking, 19-36.

doi: 10.13052/jsn2445-9739.20161.002

(c) 2016 River Publishers. All rights reserved. 
Keywords: Network resource, Software Defined Network, Service Openness, Web Technology, WebRTC.

\section{Introduction}

Evolution of the telecommunication network's architecture has made their network resources to become more available and utilizable for various network stakeholders. As the theme of the network, Internet, and Web evolves to objectify their resources and operate in software-based frame to have openness so that can have more opportunity to be used in the new environments, the network architecture and solutions have also been developed to support such changes $[1,2]$. The progress of such evolution can be described in resource accessibility-based network architectural point of view by their composition of the compounding components. In early 1990s and later, the network components were tightly coupled and connected within the network architecture with monolithic Pre-Software Oriented Architecture (SOA) like composition. Later, in 2000s, the concept of SOA was widely adapted in IT industry and the rapid expansion of Internet has brought the discussions on the reusability and openness of the network resources. Accordingly, the traditional SOA technology has been adapted to the network architecture and the compounding network components became much loosely coupled [3]. After that, with the emergence of the All IP concept, the network has evolved into the Next Generation Network (NGN) architecture and the network components are forming more decoupled compositions. Recently, such trend is more specified under the name of the Software Defined Network (SDN) and Network Function Virtualization (NFV). As the more techniques and schemes are developed for the network components to be virtualized and build with software-based composition, even the static provisioning based legacy network resources became dynamically available for services in just in time manner [4].

To this change, the rapidly enhancing improvement of the Internet and the Web has hugely affected. The changes from circuit based data transmission to the packet-based data transmission brought by the global adoption of the IP based internet protocol diminished the burden of linkage among network resources. The overriding penetration of the Web made the HyperText Transfer Protocol (HTTP) and the client-server based service architecture enough for almost every applications and services over browsers. Such environmental domination of prominent technology has simplified the network transport layer 
and adopting the concept of Web 2.0 based service architecture enabled the network components become objectified.

Nowadays, Web is not just a useful function over the telecommunication network, but an essential tool for our daily lives. As a point of contact to the cyber and online services, Web supports data portal, information search, social media service, rich multimedia content display, and so on. Recently, by utilizing the Web architectures, Web service, and Web of Device technologies, Web expands its service territory to even 'things' [5]. By doing so, Web performs the role of global data platform. The role as a global data platform is not wholly come from the development of the Web technology itself or the Web based service technology. Now, the network operators have clear role in the Information and Communications Technology (ICT) industry; as a physical and logical data infrastructure provider and manager, conducts OAM (operations, administration and maintenance) like the electric power corporations or highway corporations. It will keep the stable revenue out of doing what they have been doing. However, to get the new cash cow, it is important to looking after the new business opportunity as the Web grows in its volume and evolves in its capability while generating new service trends and requirements for existing infrastructures.

This papers studies on how to adopt the evolving trend of the network resource and service openness led by the development of the Web technology in network operator's point of view. Because the Web is the subject who requires to change the object of the resource openness from the network providers to the internet service providers. For that, this paper is composed as follows: In Chapter 2, the development of network resource open service interfaces and the state-of-the-art Web technologies are introduced as background technology. Then, the changes in the telecommunication service environment surrounding network are discussed in Chapter 3. After that, in the Chapter 4, as an use case, the deployment strategies for voice telephony over Web is described in three stages and concludes in Chapter 5.

\section{Development of Network Open Service Interfaces and Web Technology}

\subsection{History of Network Open Service Interfaces and APIs}

The network operators have expected to utilize their network resources in various ways so that can secure multiple sources of revenue. In that manner, the IT industry speedily upgraded their environmental conditions from the 


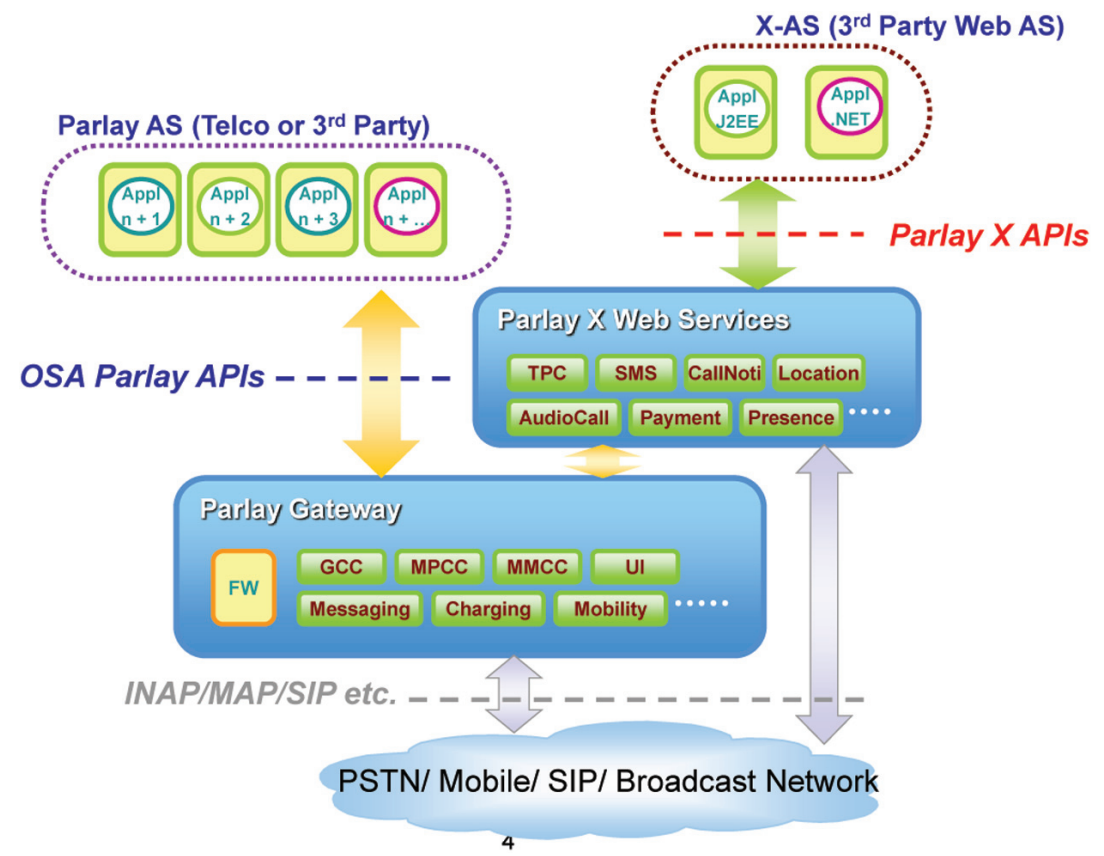

Figure 1 Parlay/OSA API and eXtended ( $3{ }^{\text {rd }}$ Party) application service.

beginning of the 21 st century. They tried to provide the network based services to have the form of easily created and deployed, executed and managed features in accordance with the network service user and provider's requirements. That intention is revealed from Parlay/OSA on the intelligence network environment to the Web services over the Web 2.0 environment and the Service Delivery Platform with the Service Oriented Architecture concept [6-8]. Figure 1 shows the concept of the Parlay/OSA based network resource utilization and the Parlay $\mathrm{X}$ based connectivity with $3^{\text {rd }}$ parties. Figure 2 demonstrates the reference architecture of the network service integration and delivery environment, which has developed in the ITU-T's standard recommendation Y.2240, "Requirements and capabilities for NGN Service Integration and Delivery Environment (NGN-SIDE)". The standard supports to open the following network resources and functions over the Next Generation Network (NGN) environment [9].

- Integration of resources from different domains over NGN (e.g. telecom domain (fixed and mobile networks), broadcast domain, internet domain, content provider domain etc.) 
- Adaptation, including abstraction and virtualization, of resources from different domains

- Resource brokering for mediation among applications and resources

- Application development environment for application developers

- Different service interfaces across ANI, UNI, SNI and NNI for exposure of NGN-SIDE capabilities and access to resources in different domains

- Mechanisms for support of diverse applications, including cloud, machine to machine, and ubiquitous sensor network applications

- Mechanisms for support of applications making usage of context based information

- Mechanisms for content management

Through the Service Delivery Platform (SDP), by offering each network resources in the form of service enablers, the network service operators have

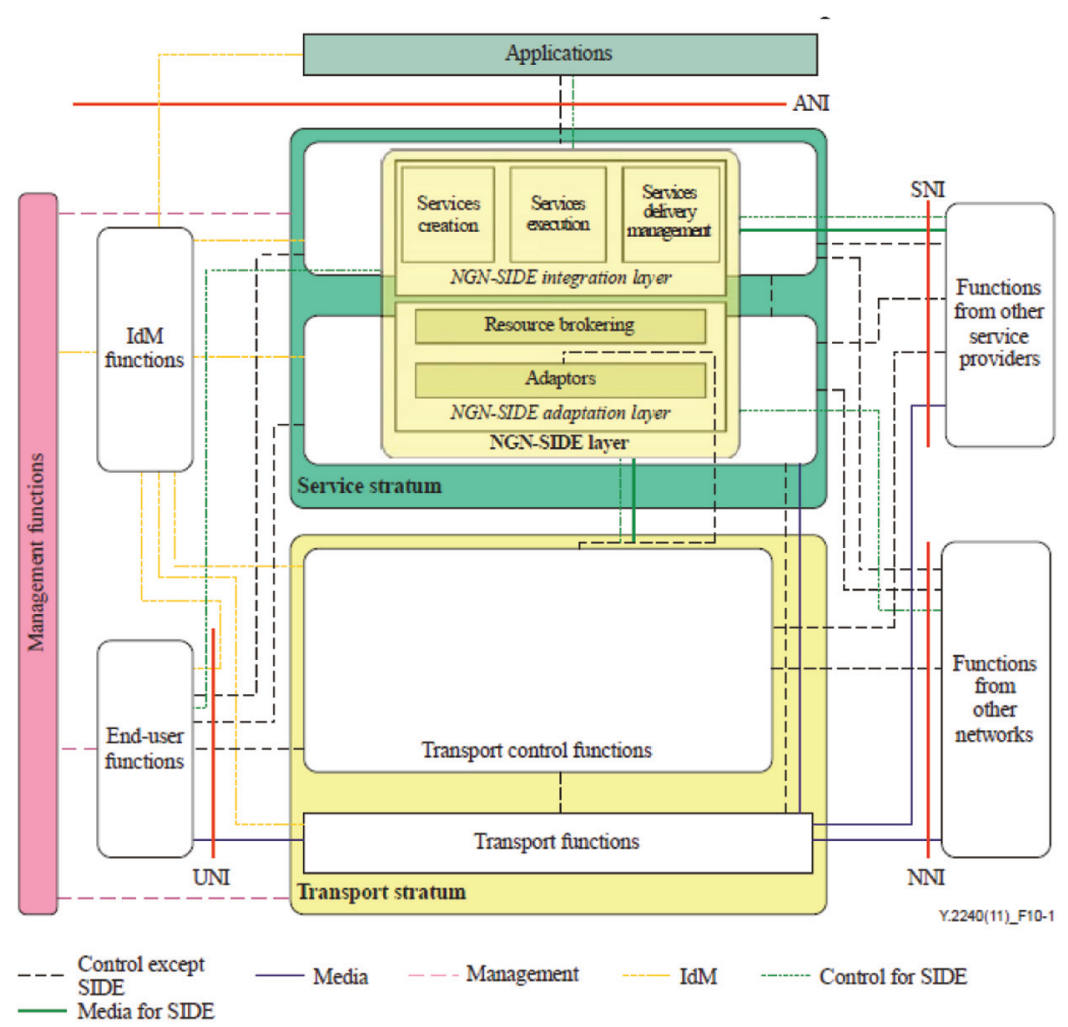

Figure 2 NGN-SIDE positioning within the NGN reference architecture. 
provided the network resource users to be able to utilize the network resources via Web service or Application Programming Interfaces (APIs).

In addition, recently, as the software-based network resource management and operation technologies, called SDN and NFV are introduced, such evolution toward network resource and service openness is even accelerating. Especially, the major features of the SDN like Programmability, Granularity, and Elasticity turned the application-awareness based service available, and embraced the opportunity of providing the capabilities on the network transport stratum, which were both not possible to be supported in the legacy network resource opening technologies [10].

\subsection{Web Technology}

As the Internet based services uses the HTTP for their services over the Web, Web is expanding its core technology from a markup-based content rendering into devices, networks, and applied services. As described below, Web has kept extending to a large convergence of software.

When it comes to the latest Web technology, W3C explains the openness of the Web as below [11]: W3C standards define an Open Web Platform for application development that has the unprecedented potential to enable developers to build rich interactive experiences, powered by vast data stores, which are available on any device. Among the several Web technologies, the HTTP 2.0 and the WebRTC plays to major role to contribute for the network resource utilization.

\subsubsection{HTTP 2.0}

Version 0.9 of HTTP was announced at 1991, HTTP/1.0 at 1996, and 1.1 at 1999. After HTTP/1.1, there were no changes on the HTTP over 10 years. However, today's web pages are about 20 times larger than 1990's in the aspects of web pages' size and number of requests and raised few issues [12].

First of all, HTTP/1.0 allowed only one request at a time on a given TCP connection. HTTP/1.1 added request pipelining, but this only addressed partially of the request concurrency problem and still has an HOL (head-ofline) blocking problem. Therefore, to achieve concurrency while reducing latency, HTTP/1.0 and HTTP/1.1 clients who desirably want to make many requests need to be able to use multiple connections to a server. Furthermore, HTTP header fields are often repetitive and lengthy. As everyone can forecast, it caused unnecessary network overhead seriously. 
The features of HTTP/2.0 to handle these issues mentioned before and to achieve better performance with low latency are as below.

- HTTP header compression

- Binary protocol

- Multiplexing

- Full-duplex interleaving and Stream priority

- Server push

\subsubsection{WebRTC}

According to the introduction of the 'webrtc.org', WebRTC is a free, open project that provides browsers and mobile applications with Real-Time Communications (RTC) capabilities via simple APIs.

WebRTC offers web application developers the ability to write rich, realtime multimedia applications (think video chat) on the web, without requiring plugins, downloads or installs. Its purpose is to help build a strong RTC platform that works across multiple web browsers, across multiple platforms.

Figure 3 is the overall architecture of WebRTC [13].

WebRTC has three APIs. The first one is MediaStream API. It represents synchronized streams of media. For example, a stream taken from camera and microphone input has synchronized video and audio tracks. Second is

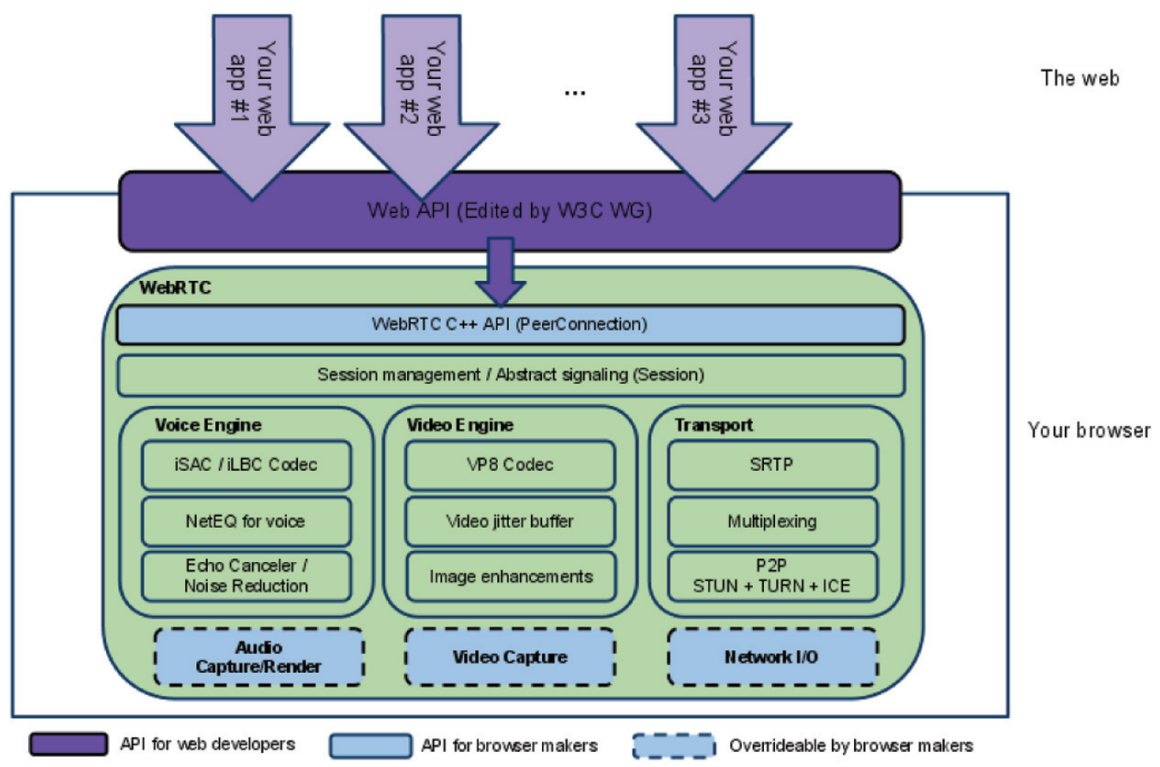

Figure 3 The overall architecture of WebRTC. 
RTCPeerConnection. It is the WebRTC component that handles stable and efficient communication of streaming data between peers. RTCPeerConnection offers easier way to access to core function without numerous complexities from underlying system. The last one is TCDataChannel. As well as audio and video, WebRTC supports real-time communication for other types of data. The RTCDataChannel API enables peer-to-peer exchange of arbitrary data, with low latency and high throughput. It can be used for gaming, remote desktop applications, real-time text chat, file transfer, and decentralized networks.

\section{Changes on Service Environment Surrounding Network}

Telco first started wired voice service on PSTN and have gradually expanded their business area to wired Internet service and mobile-based voice and data services. With the beginning of the new millennium, telco's network environment was transformed to All IP network and wired broadband. Telco then have broadened its service upon this technology advancement: from the conventional telecommunication infrastructure service to contents and media, and infra-optimized utility services. Telco got off from the simple business model and now seek to widen its value chain by all-out mobilization of their capability.

In the Telco X.0 environment, Telcos' main services are as follows: wire and wireless convergence and broadband services based on All IP Network, smart services based on contents and media, and utility services backed up by Telco infrastructure as depicted in Figure 4.

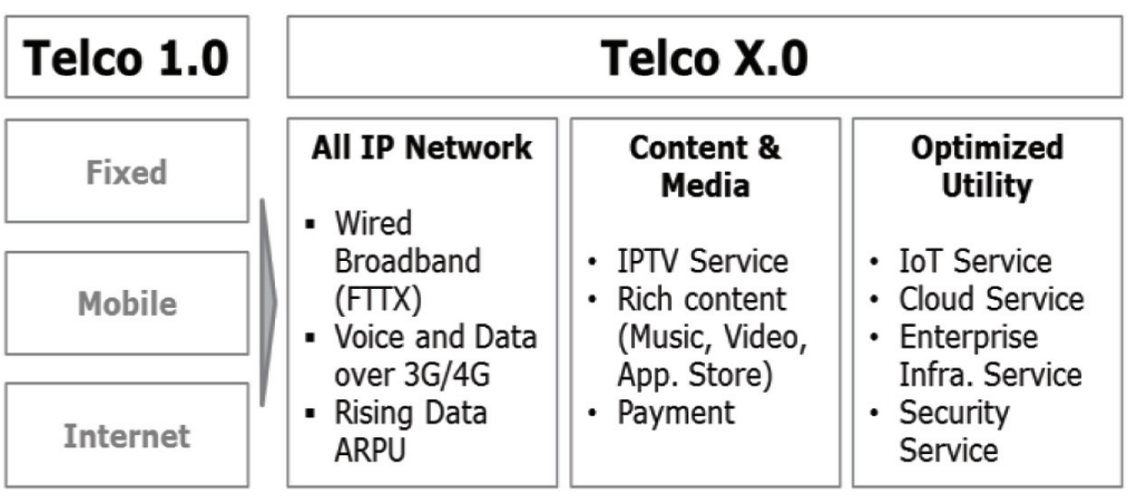

Figure 4 Value Chain Expansion via Telco X.0. 


\subsection{Optimized Utility}

As the service environment has been evolved from $\mathrm{H} / \mathrm{W}$ focused from $\mathrm{S} / \mathrm{W}$ oriented, different services utilizing the resources of Telco are introduced in the market. Cloud service and enterprise infrastructure are one type of those services, and it focuses more on providing computing resources over the network infrastructure, rather than on supplying the network itself. The end-to-end quality of network, however, needs to be assured to make this service viable. As software defined network infrastructure became widespread recently, networks are virtualized through SDN, and these resources shared and provided in the form of IaaS (Infrastructure As a Service) or PaaS (Platform As a Service).

Moreover, recent-soaring IoT (Internet of Things) services are projected to broaden the service range by connecting devices in a more accessible and universal way, which was limited in previous M2M (machine to machine) utilization.

The main reason that the convergence is called for between the Web and Telco is that the conventional models of both are in a counter position; they accordingly come to be located in a complementary relationship when it comes to provide new business zone to telecommunication service providers in Figure 5.

For Telco, abundant Internet and Web technology is required to support a high-level of Internet application for Cloud computing, future Web, and Cloud service. This Web-Telco convergence will enable Telco to take the role of major supplier of global services for both enterprises and personal users as depicted in Figure 6.

Strategic partnership between the Web and Telecommunication technology is needed, for both technologies share significant similarities and has a complementary dynamics at the same time. What Telco X.0 technology should

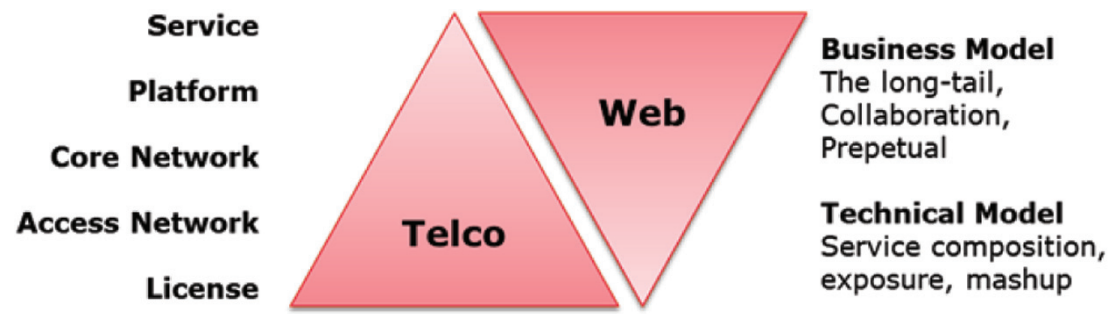

Figure 5 The opposite relationship between the conventional Telco model and the Web model. 


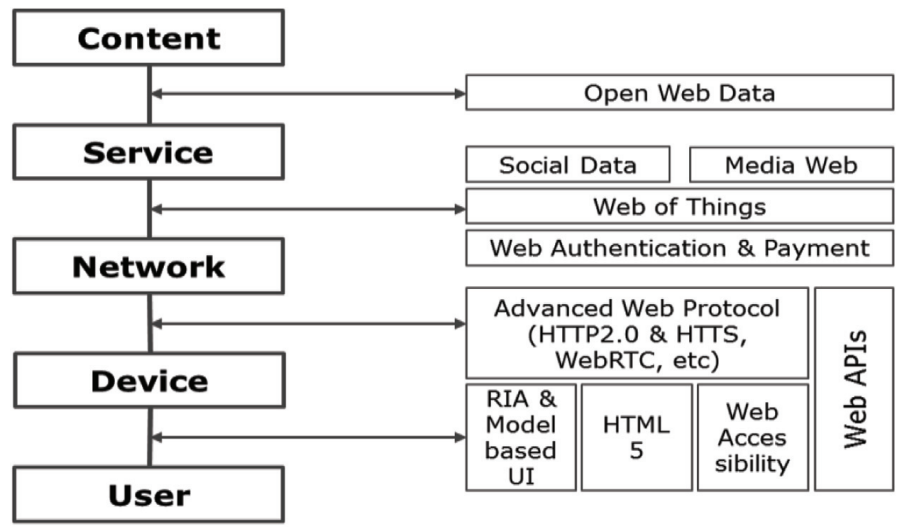

Figure 6 Overview of Web technology stack on.

pursue are, especially in the perspective of the evolution from the traditional Telco technology, two points: (1) the collaboration of content provider, who is the key player of Web 2.0, and network/communication technology provider and (2) the discovery of new business model.

Considering the major changes Telco X.0 would go through, Web technology is a must for conventional network providers, since the convergence between Web and Telecommunication tech is expected to speed up upon the

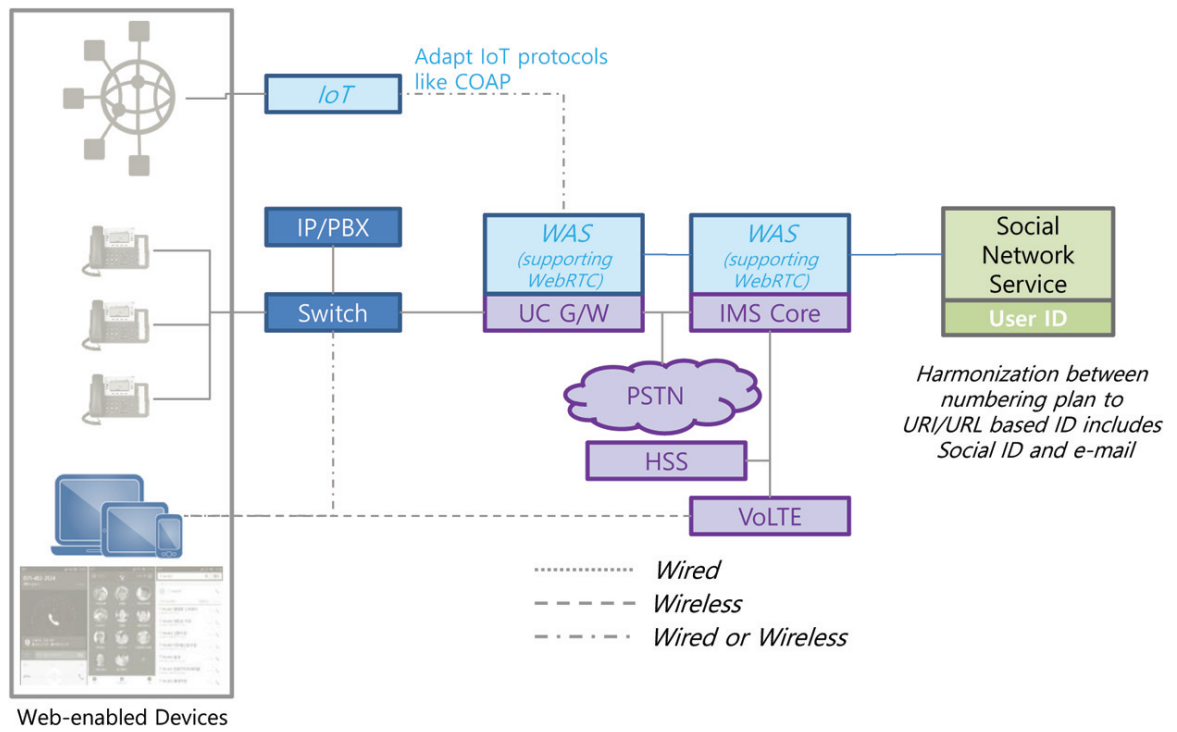

Figure 7 UCWeb evolution: stage 1 for Web-based user interfaces and unified gateway. 
complementary relationship of them. The matter is, however, who pays and who earns the money, which is difficult to be realized without the connectivity between Telco and the Web.

\subsection{Everything as a Service}

Simpler and more cost-efficient methods for doing Internet business have shown a rapid growth for the last few years. The market is moving toward an As-a-Service delivery model that provides plug-in, scalable, consumptionbased business services that deliver the business outcomes that every organization demands - increased revenue or decreased costs.

Represented as "X-as-a-Service", this model provides technical methods to utilize all the resources on-demand, real-time, and usage-based; previously, service providers had to build and manage whatever needed to provide a certain internet service.

For example, a start-up company that wants to try out a new internet business in the market formerly would have to build its own server and network, appoint corresponding managers for each task, and develop own software for its own. Nowadays, in contrast, it can use whatever resources it needs and pay the only amount accordingly, regarding the whole on-demand utility of server and network, the corresponding management, and the software application working on those infrastructures.

\section{Using Network Resources for Unified Communications over Web}

With viewpoint of telecommunication industry, the unified communication service over web (UCWeb) is the wider extension from voice over IP (VoIP) to adapt web environments. The VoIP is mainly focusing on communication protocol to deliver voice telephony service. But, the UCWeb is utilizing web technologies to deliver voice/video, IoT and many other Web-based services.

The clear feature of UCWeb is that the end users keep the web environment such as HTML and HTTP based on URL/URI. It assumes that the user device uses web-enabled screen and all the end user services are applicable through the web. The communication protocol is based on HTTP or HTTPS protocol. The display screen of end user is running on HTML.

With graceful evolution toward future web convergence, the UCWeb has to maximize the interoperability with the existing VoIP technologies. The UCWeb may use the existing VoIP protocols such as Session Initiation 
Protocol (SIP), Session Description Protocol (SDP), Real-time Transport Protocol (RTP), and Real-time Transport Control Protocol (RTCP), etc. In this case, The UCWeb can use the existing numbering scheme running on VoIP and 3G/LTE network.

According to the UCWeb evolution, the following stage from the existing wireline VoIP network may be considered.

\subsection{Using Unified Communication Gateway}

First stage is UC Gateway connecting to Web Server in Figure 8.

- The end user has two options to make voice call: one is via VoIP gateway and the others is directly connected to UCWeb server by using WebRTC. The end user can directly access the web services through WAS (web application server). The WAS offers various types of web applications by combining location and presence information. Special messaging service may be added.

- The IMS core may be included at the web server. The VoIP user may call to the customer of social network service and instance messaging services with help of WAS. Vice versa, the social customers may call the VoIP users using Social ID.

- The Internet-of-Thing (IoT) devices may be linked to web server. When the human may need to control and manage IoT devices via signalling,

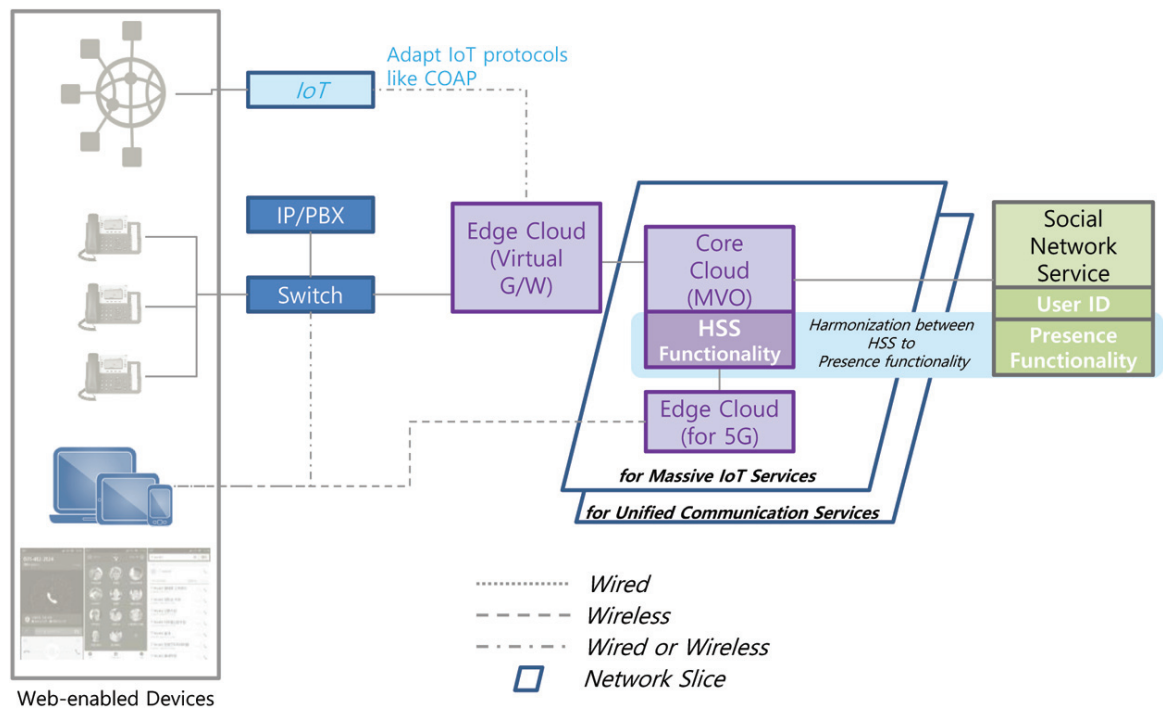

Figure 8 UCWeb evolution: stage 2 for Cloud-based Unified Communications services. 
the WebRTC protocol may be used, which is especially for IoT devices equipped with camera and speaker.

- With the extension of the existing numbering plan, the web-accessible user identification such as web ID (URL/URI), email address, and social network service SNS identifications can be used. The user ID of social network service has to be recognized by VoIP customers. The SNS users may search VoIP customers to make a call.

- The direct connection from end user to web server is relying on web standards like HTTP or Constrained Application Protocol (CoAP) etc. The WebRTC can be used to connect the web server. The WebRTC may utilize VoIP protocol to make a call.

- The emergency call or group call may also support with two options described above; via VoIP gateway or directly connect to web server. The group community call of social network services is very useful for simultaneously notification or announcement of emergency to all the members.

- The charging and billing server may connect to the web server, which may replace HSS at VoIP and 3G/LTE network in future. By combining with other web applications, various charging and billing options can be considered.

- The call-based intelligence services (e.g., call center services) can be directly connected to web server by using web APIs or the cloud concept of XaaS (anything as a service). It provides the add-on features of web services and web applications.

\subsection{Cloud-based Unified Communication Gateway}

The next stage is the Cloud-based Unified Communication Gateway replacing UC Gateway as shown in Figure 8. The roles and key features of each component in the figure are as followings:

- The core cloud (e.g., web server with presence) is a center for all the services and applications provided by Web technology. The signals in between users and devices are transmitted by HTTP 2.0 and the media information are exchanged by WebRTC.

- The end users may connect to the regional web presence server with/without the intermediate gateway and proxy system. To provide the global scalability of web presence server, the cloud-based distributed configurations of web servers are necessary to reach the billions of end users. 
- The cloud platform reaches to end users with wireline switch and/or wireless access gateway including RNC and BSC. The concept of network functional virtualization (NFV) may be used to maximize channel efficiency and avoid congestion at access network area.

- According to the migration stage, the access cloud platform may include the unified communications gateway. But, alternative signalling solutions may be chosen depending on applications.

- The mobility support is relying on the available wireline and wireless technologies. But the current 3G/LTE tunnelling and traditional VoIP protocol will be eventually upgraded. The end user device like smartphone may have some intelligence on mobility by using buffer and intelligent movement detection. Without complicate handover technique, smartphone can help the seamless connectivity by allowing some duplication or redundancy of packet streams while user moves.

- For charging and billing options, a large variety of business opportunities including sharing economy may be coming at near future. The traditional home subscriber system (HSS) may be upgraded in order to offer plentiful business solutions.

\section{Conclusion}

The network resource and service openness and softwarization have been progressively developed in both technology and architectural manner. Recently, it is showing even widened coverage which is from the network application level (L7) to the data link level (L2) via the state-of-the-are researches on SDN and NFV. Along with that, the Web technology has also been steadily developed to utilize the new resources. Evolved from the early form of the link among web pages, current Web technology is applied in a wide range of services: sensor/device, service/application, and cloud. As with the giant leap of Web-based media (social media, video portal, etc.), the Web is now essential for people's daily life.

This paper looks out the evolution of the two technologies and provides a blueprint on the enhanced communication service architecture using WebRTC over the SDN and NFV based upcoming network environment. The network, the core asset of Telco which gives the grounds for its business, will evolve to be functionalized via SDN/NFV and if so, software will be at the center of network control. Network provider may amplify the Web leverage by utilizing resources in a lighter and smaller unit, which is achievable with the adoption of microservice-based software architecture and DevOps. Therefore, further 
study on this area is required to enhance its cost-effective applicability in current network.

\section{References}

[1] Chung, J. Y., Jeong, T., Yoo, J.-H., Hong, J. W.-K. (2015). "Define interfaces for network softwarization," in Proceedings of KICS, 870-871.

[2] Shin, M.-K., Nam, K.-H., Kim, H.-J. (2012). "Software-defined networking (SDN): a reference architecture and open APIs," in 2012 International Conference on ICT Convergence (ICTC), 360-361.

[3] Magedanz, T., Blum, N., and Dutkowski, S. (2007). Evolution of SOA concepts in telecommunications. Computer 40, 46-50.

[4] Aslan, M. and Matrawy, A. (2016). On the impact of network state collection on the performance of SDN applications. Commun. Lett. IEEE 20, 5-8.

[5] W3C Web of Device. Available at http://www.w3.org/standards/webofdev ices/

[6] W3C Web Service Architecture. Available at https://www.w3.org/TR/ws$\operatorname{arch} /$

[7] Bennett, A. J., Grech, M. L. F., Unmehopa, M. R., and Vemuri, K. V. (2003). Service mediation standards. Bell Labs Tech. J. 7, 77-90.

[8] Qiao, X., Chen, J., Gu, P., and Li, Y. (2014). Opening up telecom networks with a lightweight web element service cloud for ordinary users in the web 2.0 era. IEEE Commun. Mag. 52, 127-133.

[9] ITU. (2011). Requirements and Capabilities for NGN Service Integration And Delivery Environment (NGN-SIDE), ITU-T Recommendation Y.2240.

[10] Jarschel, M., Zinner, T., Hossfeld, T., Tran-Gia, P., and Kellerer, W. (2014). Interfaces, attributes, and use cases: A compass for SDN. IEEE Commun. Mag. 52, 210-217.

[11] W3C Standards. Available at http://www.w3.org/standards/

[12] Average Web Page Breaks 1600K. Available at http://www.websiteoptimi zation.com/speed/tweak/average-web-page/

[13] WebRTC. Available at http://webrtc.org 


\section{J. Yang et al.}

\section{Biographies}

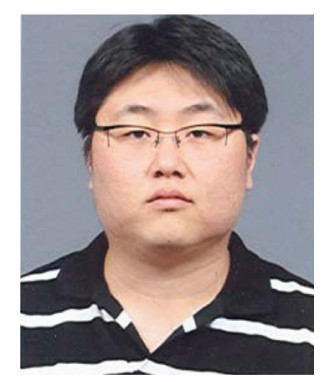

J. Yang (S'05) received M.S. in computer science from InJe University in 2005 and HERIT Inc. in 2008 and currently he is Ph.D. candidate student in Korea Advanced Institute of Science and Technology (KAIST). His main research interests include next generation network, multimedia streaming issues, and future media.

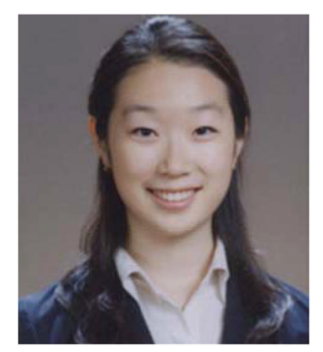

H. Park (S'07) received M.S. in communications engineering from Information and Communications University in 2007 and currently, she is Ph.D. candidate student in Korea Advanced Institute of Science and Technology (KAIST). Her main research interests include IPTV, broadcast networks, multimedia streaming issues, and next generation network. 


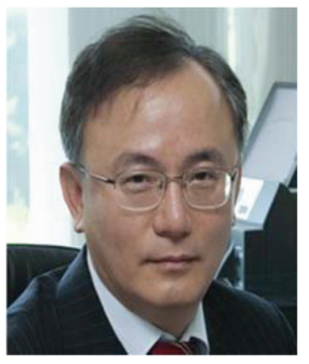

J. K. Choi (M'88-SM'00) received the B.Sc. (Eng.) from Seoul National University in electronics engineering, Seoul, Korea in 1982, and M.Sc. (Eng.) and Ph.D. degree in 1985 and 1988, respectively, in electronics engineering from Korea Advanced Institute of Science and Technology (KAIST). From June 1986 until December 1997, he was with the Electronics and Telecommunication Research Institute (ETRI). In January 1998, he joined the Information and Communications University (ICU), Dae jeon, Korea as Professor. At year 2009, he moves to Korea Advanced Institute of Science and Technology (KAIST) as Professor. He is a Senior Member of IEEE, the executive member of The Institute of Electronics Engineers of Korea (IEEK), Editor Board of Member of Korea Information Processing Society (KIPS), Life member of Korea Institute of Communication Science (KICS). 
